\title{
LONG-TERM FOLLOW-UP IN TUMORAL ARTHROPLASTY
}

\author{
doi: 10.2478/rojost-2018-0065

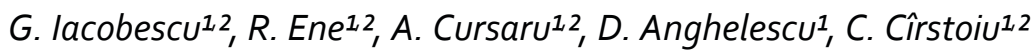 \\ 1 University Emergency Hospital, Bucharest, Romania \\ 2"Carol Davila" University of Medicine and Pharmacy, Bucharest, Romania
}

Introduction. Efficient management of a segmental resection or major osteolysis in the distal femur secondary to a tumor formation remains a controversial problem. Available options include the use of a modular or customized megaprosthetic implant. Modularity allows versatility for reconstruction and avoids the delay required to make a customized implant.

Hypothesis and type of study. Performing a clinical and radiological retrospective study that aims to evaluate long-term efficiency in the use of megaprostheses in segmental distal femur resections. Elaboration of patient selection criteria for modular prosthesis.

Materials and methods. We followed retrospectively 33 patients for 5 years from the time of the first surgery.

We evaluated the implant stability, the late complications rate, and the long-term functional recovery of patients with distal femoral tumors who underwent segmental resections and subsequently reconstructive arthroplasty.

Results. Thirty of the 33 patients maintained a mobile knee joint. An intermediate staging was performed at 30 months, which determined tumor recurrence in 2 patients, aseptic degradation of the components in 3 of them, and septic degradation in two of the evaluated cases. Because a tumoral recurrence occurred on the $45^{\text {th }}$ month, the need for amputation of the prosthetic limb was imposed. The degradation of the polyethylene component (in 5 cases) was observed in the 5 -year assessment.

The functional results were excellent with the Musculoskeletal Tumor Society Score of $88 \%$ and a Toronto Extremity Severity Scale Score of 94\%.

Conclusions. Patients with distal femoral bone tumors undergoing modular reconstruction prosthetic arthroplasty have excellent functional results with retaining the affected limb and knee mobility. There was a close correlation between correctly applying the selection criteria for patients undergoing prosthesis intervention and functional recovery results.

Keywords: megaprostheses, distal femoral tumor, reconstructive arthroplasty 